\title{
Evidence that ethanol selectively alters dopamine and serotonin metabolism as well as peptidergic levels in CA3 hippocampus of spontaneously alcohol preferring rats
}

\author{
Francesco Crespi*, Francesco Congestri, Francesca Formenti \\ Department of Biology, GSK Verona, Italy
}

\begin{abstract}
Ethanol is an abused psychoactive drug and alcohol abuse is formally recognized as pathology. Alcohol interacts with several central neurotransmitter systems, among others dopamine (DA) serotonin (5-HT) and peptides such as cholecystokinin (CCK).

Voltammetry is an electrochemical methodology for direct in situ and real time measurement of electronically active chemicals. In particular differential pulse voltammetry (DPV) associated with micro biosensors such as specifically treated carbon fiber micro electrodes (mCFE) has been shown able to measure dopamine (DA) and serotonin (5-HT) metabolism as well as CCK levels in discrete brain areas of rodents.

In the present work the DPVoltammetric levels of DA and 5-HT metabolism as well as those of the peptidergic signal related to CCK are monitored in the frontal cortex (FR1), the caudatelo putamen, the nucleus accumbens and the hippocampus, in particular the CA3 region, of spontaneous alcohol drinking rats (AD rats) and compared to those gathered in water preferring rats (WP rats). These regions have been selected as they are implicated in the drug dependence phenomena and AD rats as well as WP rats have been obtained within naïve rats submitted to free choice between water and alcohol.

Results show that changes of DA and 5-HT metabolism and peptidergic (CCK) levels occurred selectively in the CA3 hippocampus of AD rats, suggesting that this region is particularly sensitive to alcohol.

Since these data have been obtained in adult rats it may be possible to suggest that the hippocampal functions are selectively altered by alcohol not only in adolescents as proposed earlier but also in adults. This may be useful indication for the development of innovative pharmacological approaches towards alcohol abuse in both adolescent and adult addicted.
\end{abstract}

\section{Introduction}

Alcohol is the second most widely abused psychoactive drug after caffeine. In 1990, the American Medical Association formally recognized alcohol abuse as pathology. In the aim to develop new drugs for the treatment of alcohol abuse, recent studies have shown that ethanol interacts with several central neurotransmitter systems, such as:

- The GABAergic system activity via increasing the number of GABA receptors $[1,2]$;

- The dopaminergic system: resulting either in increase $[3,4]$ or decrease $[5,6]$;

- The serotoninergic system that seems to play a role in the control of ethanol intake [5,7];

- The CCK system with the evidence of the development of preference for ethanol in naïve rats when treated with CCK receptor antagonists $[8,9]$

- The NO system: where ethanol, at pharmacologically significant doses, strongly inhibits striatal $\mathrm{NO}^{\bullet}$ production and release apparently through inhibition of NMDA receptor function. Inhibition of NMDA receptor-mediated activation of the NO• pathway could be a primary neurobiological mechanism contributing to the effects of ethanol [10].
Voltammetry is an electrochemical methodology for direct in situ and real time measurement of electronically active chemicals without the need for sample preparation or chromatographic analysis.

Especially DPV associated with micro biosensors and in particular with specifically treated carbon fiber micro electrodes (mCFE) 7 to 30 micrometer diameter (Figure 1) is an electrochemical methodology that allows monitoring specific compounds in the extracellular fluid of discrete brain areas [11]. This methodology complies with the majority of the conditions required for examining specific compounds in the extracellular fluid [12]. Here DPV has been performed in various brain areas of rats submitted to free choice between water and alcohol as described earlier (13). In particular the levels of DOPAC (DA metabolite), 5-HIAA (serotonin metabolite) and the levels of the peptidergic signal related to CCK [13-16] are monitored in the Frontal cortex (FR1) the caudatelo putamen, the nucleus accumbens and the

${ }^{\star}$ Correspondence to: Francesco Crespi, Department of Biology, GSK Verona, Italy, Tel: + 39 3481452720; E-mail: fm.crespi@libero.it

Key words: alcohol, free choice, voltammetry, rat brain, dopamine, serotonin, cholecystokinin

Received: January 01, 2019; Accepted: January 21, 2019; Published: January 24, 2019 
Crespi F (2019) Evidence that ethanol selectively alters dopamine and serotonin metabolism as well as peptidergic levels in CA3 hippocampus of spontaneously alcohol preferring rats

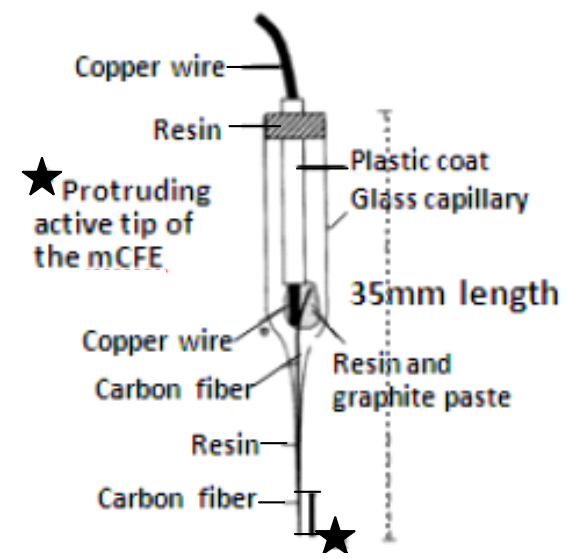

$0.2 / 0.3 \mathrm{~mm}$
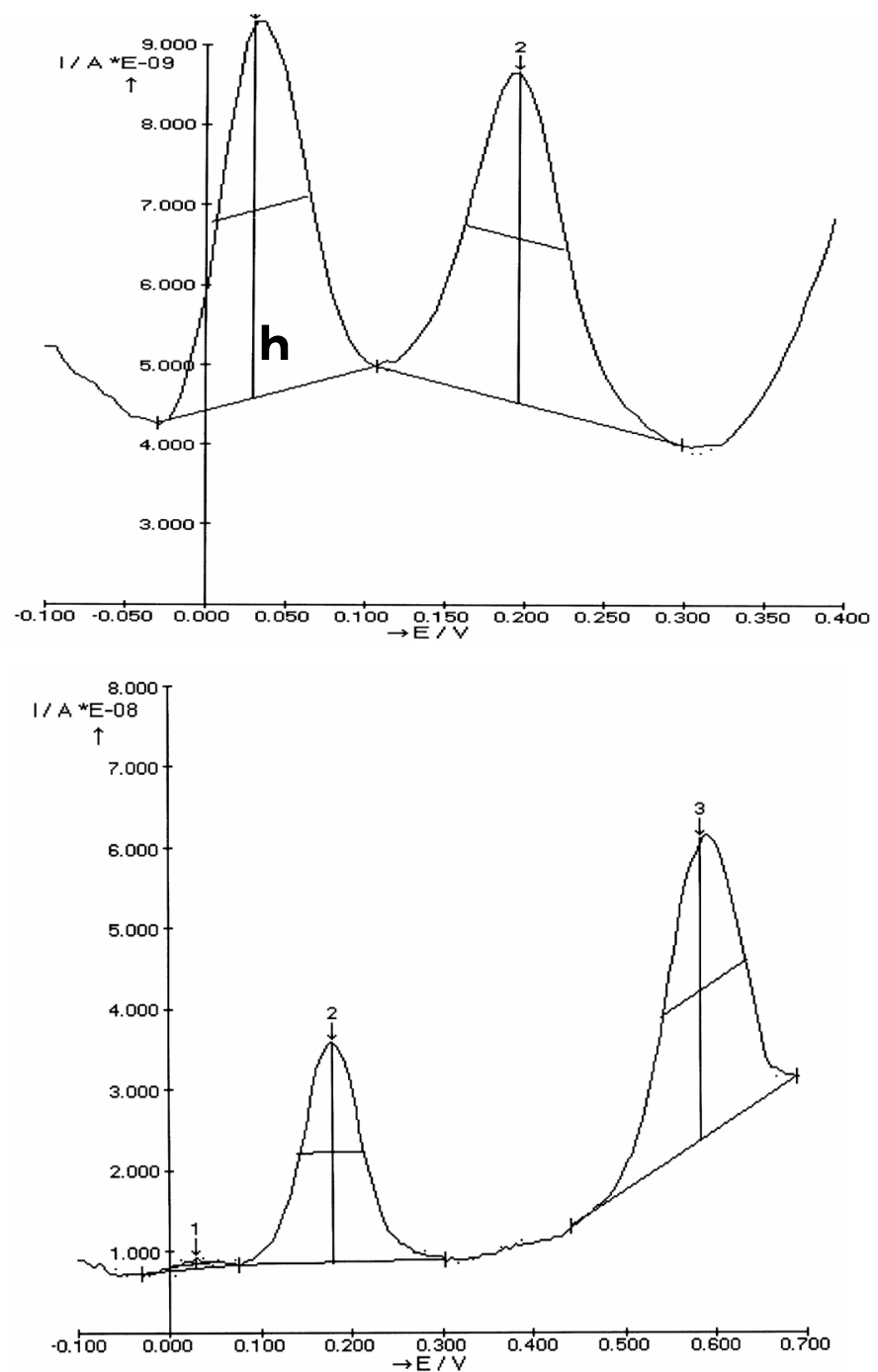

Figure 1. Top: Schematic representation of the micro-biosensor (mCFE) used here. The protruding active tip (approximately 200/300 $\mu \mathrm{m}$ length) is previously treated as described in Methods Middle: Typical in vitro DPV scan obtained with the mCFE when immersed in a PBS solution at $\mathrm{pH} 7.4$ containing a mixture of DOPAC, $50 \mu \mathrm{M}$ and $5 \mathrm{HIAA}, 25 \mu \mathrm{M}$. It results in two distinct oxidation peaks at approximately $30 \mathrm{mVolts}$ and $200 \mathrm{mVolts}$, respectively (X axis).

h: size of the peak measured in nanoAmperes (Y axis)

Bottom: Typical in vitro DPV scan obtained with the mCFE immersed in a PBS solution at pH 7.4 containing a mixture of 5-HIAA, $25 \mu \mathrm{M}$ and CCK $50 \mu \mathrm{M}$. It results in two distinct oxidation peaks at approximately $200 \mathrm{mVolts}$ and $600 \mathrm{mVolts}$, respectively

The measurements of oxidation potential (X axis) and of oxidation current (Y axis) are done automatically by the Autolab polarograph (potentiostat/galvanostat Ecochemie, The Netherlands) linked to an IBM pc computer equipped with a general purpose electrochemical system software (GPES) package 
Crespi F (2019) Evidence that ethanol selectively alters dopamine and serotonin metabolism as well as peptidergic levels in CA3 hippocampus of spontaneously alcohol preferring rats

hippocampus, in particular the CA3 region. These regions have been selected as they are implicated in the drug dependence phenomena [17-22].

DPVoltammetric data gathered indicate a selective significant change on the levels of catecholaminergic and serotonergic metabolism as well as those of the peptidergic signal in CA3 of hippocampus, while no significant alterations of the above mentioned voltammetric signals have been detected within the other brain regions analyzed.

\section{Methods}

DPVoltammetry is applied via a three-electrode potentiostat system as described previously $[11,12,14]$. The mCFE were prepared using a $12 \mu \mathrm{m}$-diameter carbon fiber (Carbone Lorraine, Lyon, France) with an electro-active tip of $0.2 / 0.3 \mathrm{~mm}$ length and were electrically treated firstly with a voltage from zero to 3 Volts, $70 \mathrm{~Hz}, 10 \mathrm{~s}$, then with continuous potentials $(+1.5$ Volts, $5 \mathrm{~s}$ and -0.9 Volts, $5 \mathrm{~s})$. The electrochemical treatment as well as the successive in vitro calibration of the mCFE were carried out with the auxiliary, reference, and working electrodes immersed in $0.1 \mathrm{M}$ phosphate-buffered saline (PBS) at $\mathrm{pH} 7.4$ [14-16]. The electrochemical treatment enables the detection of three separate peaks in vitro in a solution of ascorbic acid $5 \mathrm{mM}$; DOPAC, $50 \mu \mathrm{M}$; and 5-HIAA, $25 \mu \mathrm{M}$, as already demonstrated $[11,14]$. Furthermore, it also allows the in vitro detection of a further oxidation peak when the DPV recordings were made in the same solution with the addition of amino acids or peptides $[15,16]$ and when the DPV scan rate used was $10 \mathrm{mV} / \mathrm{s}$ from -250 to $+950 \mathrm{mV}$ at a step size of $50 \mathrm{mV}$ (Figure 1).

The DPV measurements of oxidation potentials and of oxidation currents are done automatically by the Autolab polarograph (potentiostat/galvanostat Ecochemie, The Netherlands) linked to an IBM pc computer equipped with a general purpose electrochemical system software (GPES) package.

\section{Animals}

Twenty male adult rats (Wistars, 220-250 g) were supplied by Charles-River (Italy) and kept in temperature- and humiditycontrolled rooms $\left(22^{\circ} \mathrm{C}, 50 \%\right)$. All animal procedures were carried out in accordance with the Italian law (Legislative Decree no. 116, 1992) which acknowledges the European Directive 86/609/EEC and were fully compliant with the GlaxoSmithKline policy on the care and use of laboratory animals and codes of practice. Furthermore, all efforts were made to minimize the number of animals and their suffering.

\section{Behaviour}

In the first week of adaptation to alcohol, animals received ethanol $2 \%(\mathrm{v} / \mathrm{v})$, solution known not to be selective [23,24]. Successively animals were submitted to a free choice between a bottle of fresh water and a bottle of an ethanol solution at a concentration $(10 \% \mathrm{~V} / \mathrm{V})$ that has been shown allowing selecting between alcohol drinking rats (AD rats) or water preferring rats (WP rats) $[13,23,24]$. For details of the drinking free choice procedure used here see in particular ref 13.

\section{Ex vivo DPV}

At the conclusion of the drinking behaviour, rats were sacrificed, the brain rapidly removed, and brain slices were prepared and then submitted to DPV measurements as described earlier [13,25].

\section{Statistical Analysis}

Row data were subjected to ANOVA, with comparison between
WP rats and AD rat's values performed using the Bonferroni (Dunn's) test. Then, the results were presented as $\%$ of control values, mean \pm s.e.m., ${ }^{\star} \mathrm{p}<0.05$.

\section{Results}

\section{Drinking Behaviour}

During the week of adaptation, the intake of $2 \%$ ethanol (v/v) was similar in all animals. Successively, when submitted to the free choice between water and a solution of ethanol $10 \% \mathrm{v} / \mathrm{v}$, it was possible to obtain rats that consumed a significant amount of ethanol $10 \% \mathrm{v} / \mathrm{v}$. These were called alcohol drinking (AD) rats, and were about $40 \%$ of the animal population tested. This occurred within 4 weeks, when the AD rats had reached a regular consumption of $15.2 \pm 3.4 \mathrm{ml}$ ethanol $10 \%$ $\mathrm{v} / \mathrm{v}$ daily (mean \pm s.e.m). The remaining rats, so called water preferring (WP) disliked consuming alcohol as they ingested approximately only $3.2 \pm 1.1 \mathrm{ml}$ water, daily (mean \pm s.e.m) (Figure 2 ).

\section{DPVoltammetry}

Figure 3 shows typical DPVoltammograms obtained i.e. in the slice preparations from AC3 hippocampus. Four major oxidation signals
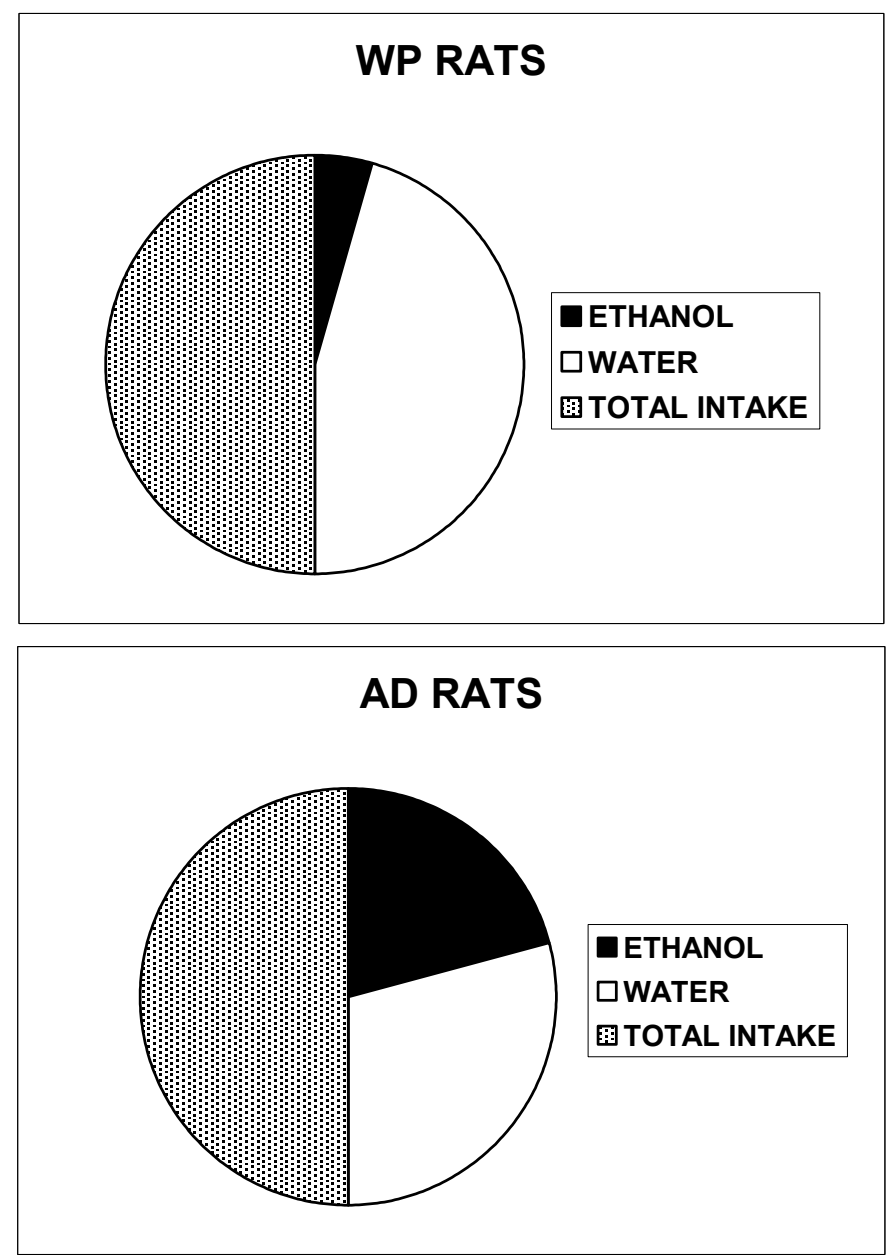

Figure 2. Total amount (ml) of water AND ethanol $10 \% \mathrm{v} / \mathrm{v}$ consumed daily by rats (WP or AD) when submitted to the free choice between 1 bottle of water AND 1 bottle of ethanol 10\% $\mathrm{v} / \mathrm{v}$. ([13] for the details of the behavioral alcohol and WATER drinking free choice procedure) WP rats: $3 \pm 1 \mathrm{ml}$ ethanol $10 \% \mathrm{v} / \mathrm{v}$ and $31 \pm 4 \mathrm{ml}$ water.

AD rats: $15 \pm 3 \mathrm{ml}$ ethanol $10 \% \mathrm{v} / \mathrm{v}$ and $21 \pm 5 \mathrm{ml}$ water.

Data are expressed as liquid consumption, SEM are omitted for clarity 
Crespi F (2019) Evidence that ethanol selectively alters dopamine and serotonin metabolism as well as peptidergic levels in CA3 hippocampus of spontaneously alcohol preferring rats

(peaks) are obtained using the Autolab apparatus (Ecochemie, the Netherlands) that measure automatically the size of each peak. The size (h: measured in nanoAmperes) gives the direct measure of each current signal while in abscissa is indicated the specific oxidation potential.
In this figure, the top voltammogram is obtained in the CA3 slice of a WP rat, while the bottom one in the CA3 slices of an AD rat. Similar data are obtained in the other WP or AD rats, (Figure 4). Note in the latter the decrease of the current size of Peaks 2 and 3, corresponding
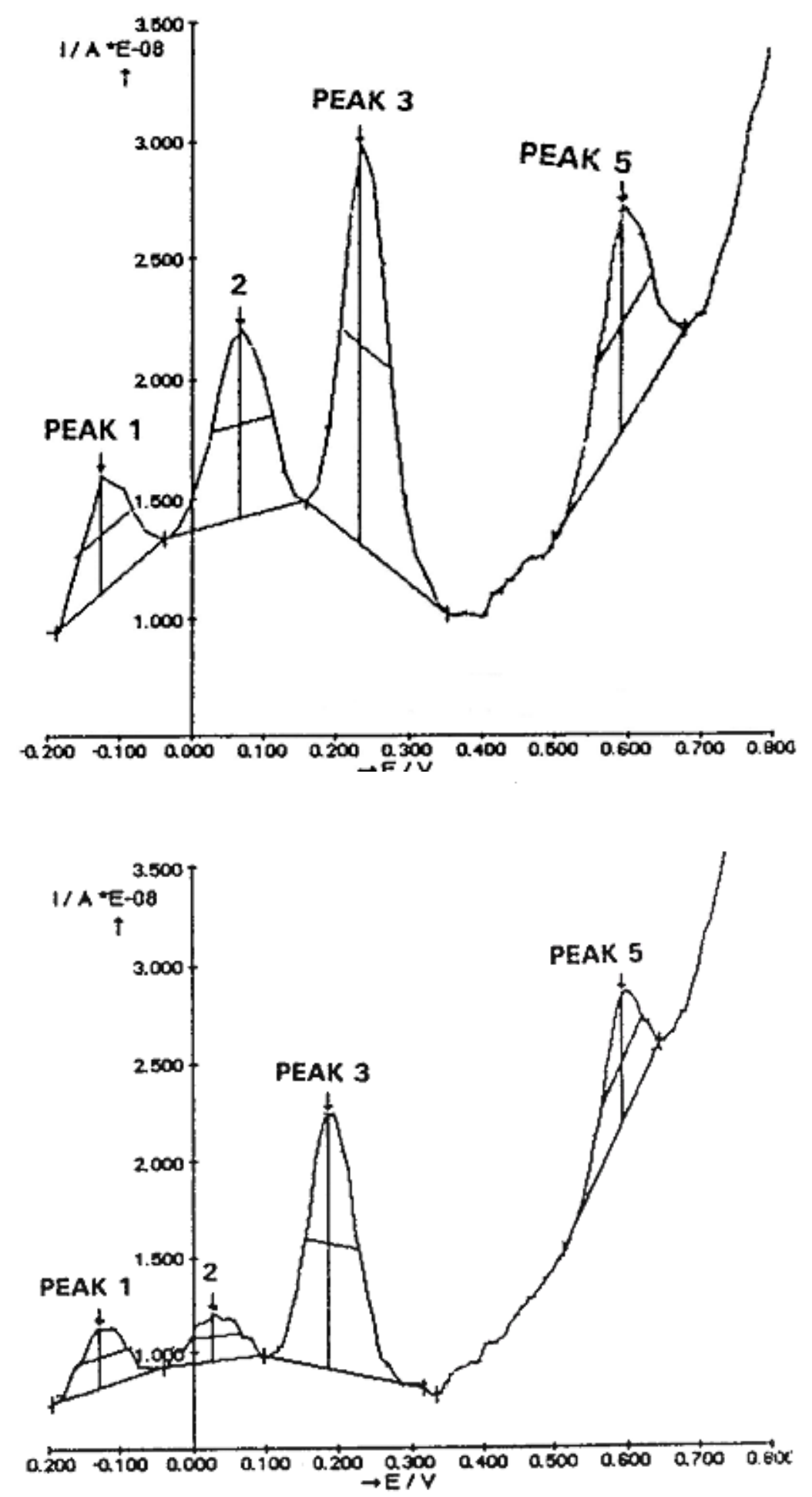

Figure 3. Typical ex vivo DPVoltammograms obtained i.e. in the CA3 hippocampus with the mCFE: TOP in one WP rat, BOTTOM in one AD rat

Note the automatic detection by the Autolab - GPES package of four peaks, corresponding to oxidation of ascorbic acid (peak 1), DOPAC (peak 2), 5-HIAA (peak 3) and CCK related signal (Peak 5) $[11,16]$. At approximately $400 \mathrm{mV}$ there is a non-significant alteration of the current baseline that may correspond to the so called peak 4 recorded i.e. in the striatum and corresponding to the oxidation of 3-methoxytyramine $[29,30]$. 

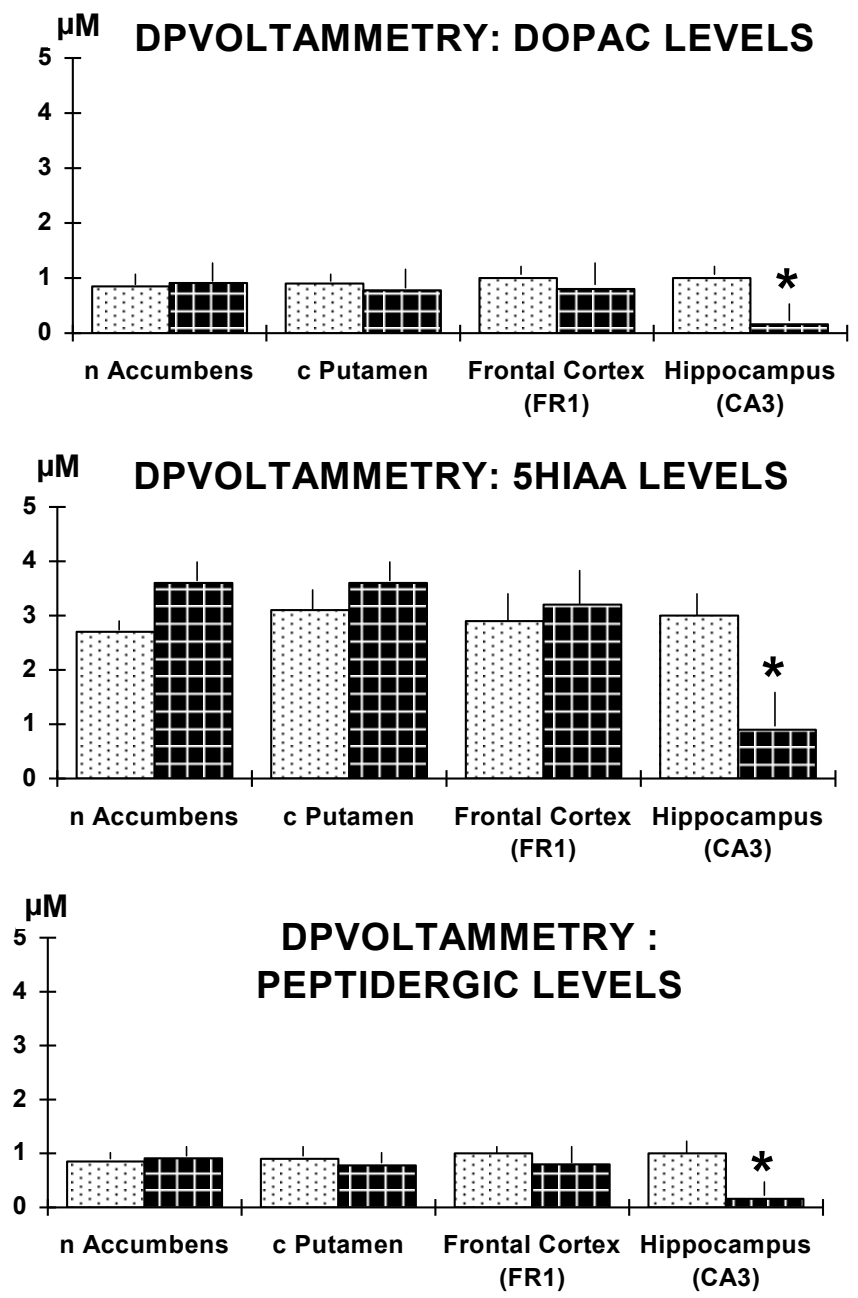

Figure 4. Results obtained in brain slices of the four regions selected. Note the significant decrease of the current size of the DPVoltammetric signals corresponding to the oxidation of DOPAC and 5-HIAA, respectively as well as of that corresponding to the oxidation of the peptidergic CCK-like DPV peak [11,13].

Data are expressed in $\mu \mathrm{M}$ levels, mean \pm sem. ${ }^{*} \mathrm{p}<0.05$.

to the oxidation of DOPAC and 5-HIAA, respectively as well as that of Peak 5, corresponding to the oxidation of CCK $[11,13]$.

\section{Discussion}

It has been widely observed that alcohol impacts brain function and behaviour, and possibly adolescents seem to be more vulnerable than adults to impairments following repeated alcohol exposure [26]. Studies indicate that alcohol alters learning and memory by enduringly damaging the hippocampus and in particular the CA3 hippocampal subfield [17]. Again, behavioral as well as extra-cellular and whole-cell electrophysiological studies to evaluate the impact of acute and chronic ethanol exposure indicated clearly that hippocampal function is specifically responsive to ethanol during development [18]. However, the mechanism of action of alcohol is poorly understood [17].

Studies in rodents and in humans show that alcohol stimulates dopamine release mostly in the striatum and in the nucleus accumbens: occurrence that is implicated in the reinforcing effects of drugs of abuse $[19,20]$.

The frontal cortex is also implicated in the context of drug (alcohol) addiction and in particular it has been shown that it regulates the value of rewards by modulating dopamine increases in the ventral striatum [21].
In addition, microdialysis experiments performed to monitor the extracellular concentrations of dopamine, serotonin, and their major metabolites DOPAC and 5-HIAA, respectively in the nucleus accumbens of alcohol-naive rats selectively bred high-alcohol-drinking (HAD) and low-alcohol-drinking (LAD) demonstrated that in both HAD and LAD groups, the extracellular levels of DA were increased following the intra-peritoneal injection of 1.0 and $2.0 \mathrm{~g}$ of ethanol $/ \mathrm{kg}$ body weight while only the $2.0 \mathrm{~g} / \mathrm{kg}$ dose elevated the concentration of 5-HT. It appeared also that none of the ethanol doses altered the extracellular levels of their major metabolites DOPAC and 5-HIAA [22].

The frontal cortex is also implicated in the context of drug (alcohol) addiction and in particular it has been shown that it regulates the value of rewards by modulating dopamine increase in the ventral striatum [21].

Accordingly, in the present work, the DPVoltammetric data gathered in the brain regions analyzed i.e. frontal cortex, nucleus accumbens (nAccumbens) and striatum (c.putamen) of alcohol drinking $(\mathrm{AD})$ rats show no significant changes on the levels of DA and 5-HT metabolites DOPAC and 5-HIAA.

In contrast, DOPAC and 5-HIAA levels appeared significantly decreased in the CA3 hippocampus of the $\mathrm{AD}$ rats versus WP rats, thus supporting the proposed selective influence of alcohol upon this brain area [18].

It has been shown that CCK, in addition to its wide distribution in the cerebral cortex, is present in abundance in many areas of the hypothalamus and limbic system, and in particular in the CA3 region of the hippocampus $[27,28]$.

The feasibility of monitoring neuropeptides in vitro and in vivo by means of DPVoltammetry has been reported $(11,15,16)$ and it has been shown that the peptidergic signal occurring at the oxidation potential of approximately $+600 \mathrm{mV}$ corresponds to the oxidation of CCK $[8,13]$.

Again, here it is shown that this peptidergic signal is significantly and specifically decreased in the CA3 hippocampus of AD rats versus WP rats.

This is possibly in accord with the previously reported evidence that peptides more than biogenic amines are involved in the free choice of drug intake suggesting a putative influence of these compounds upon the phenotype involved in the preference for drugs of abuse. This may be a significant observation in the contest of the development of a novel pharmacological approach to interfere with such phenotype in the attempt to reduce the willingness to consume drugs.

Furthermore, since the animals involved in the present experiments are adult rats it may be possible to suggest that the hyppocampal functions are selectively altered by alcohol not only in adolescents $[17,18]$ but also in adults. Again, this observation may help in the design of innovative pharmacological approaches towards alcohol abuse in both adolescent and adult addicted.

\section{References}

1. Ticku MK, Lowrimore P, Lehoullier P (1986) Ethanol enhances GABA induced 36Clinflux in primary spinal cord cultured neurons. Brain Research Bullettin 17: 123-126.

2. Roberto M, Madamba SG, Moore SD (2003) Ethanol increases GABAergic transmission at both pre- and postsynaptic sites in rat central amygdala neurons. PNAS 100: 2053-2058.

3. Di Chiara G, Imperato A (1986) Alcohol and barbiturates preferentially stimulate dopamine release in the nucleus Accumbens of freely moving rats. Symposia in Neuroscience. Fidia Research Series 3: 89-96. 
Crespi F (2019) Evidence that ethanol selectively alters dopamine and serotonin metabolism as well as peptidergic levels in CA3 hippocampus of spontaneously alcohol preferring rats

4. Di Chiara G, Bassareo V, Fenu S, De Luca MA (2004) Dopamine and drug addiction: the nucleus accumbens shell connection. Neuropharmacol 4: 227-24.

5. Gessa GL, Colombo G, Fadda F (1990) [Experimental models of alcoholism]. Recenti Prog Med 81: 162-165. [Crossref]

6. Ma H, Zhu G (2014) The dopamine system and alcohol dependence. Shanghai Arch Psychiatry 26: 61-68. [Crossref]

7. Johnson BA (2004) Role of the serotonergic system in the neurobiology of alcoholism: implications for treatment. CNS Drugs 18: 1105-1118. [Crossref]

8. Crespi F (1998) The role of cholecystokinin (CCK), CCK-A or CCK-B receptor antagonists in the spontaneous preference for drugs of abuse (alcohol or cocaine) in naive rats. Methods Find Exp Clin Pharmacol 20: 679-697. [Crossref]

9. Crespi F, Corsi M, England T, Ratti E, Trist DG, et al. (1997) Spontaneous preference for ethanol in naive rats is influenced by cholecystokinin A receptor antagonism. Alcohol 14: 327-332. [Crossref]

10. Rossetti Z, Crespi F (2004) Inhibition of Nitric Oxide release in vivo by ethanol Alcoholism: Clinical and Experimental Research 28: 1-6.

11. Crespi F (1990) In vivo voltammetry with micro-biosensors for analysis of neurotransmitter release and metabolism. J Neurosci Methods 34: 53-65. [Crossref]

12. Crespi F (2013) Serotonin, how to find it. J Malta Chamb Sci pp. 14-22.

13. Crespi F (1998) The role of cholecystokinin (CCK), CCK-A or CCK-B receptor antagonists in the spontaneous preference for drugs of abuse (alcohol or cocaine) in naive rats. Methods Find Exp Clin Pharmacol 20: 679-697.

14. Crespi F (1991) In vivo voltammetric detection of neuropeptides with micro carbon fiber biosensors: possible selective detection of somatostatin. Anal Biochem 194: 6976. [Crossref]

15. Crespi F (2017) Central [CNS] and Peripheral [Gastric Tissue] Selective Monitoring of Somatostatin (SRIF) with Micro-Sensor and Voltammetry in Rats: Influence of Growth Factors (GH, EGF). Biosensors 7: 53-63.

16. Crespi F, Sharp T, Maidment N, Marsden CA (1984) Differential Pulse Voltammetry: simultaneous in vivo measurement of ascorbic acid, catechols and 5-hydroxyindoles in the rat striatum using a single carbon fibre electrode. Brain Research 322: 135-138.

17. Zucca S, Valenzuela CF (2010) Low Concentrations of Alcohol Inhibit BDNFDependent GABAergic Plasticity via L-type Ca2+ Channel Inhibition in Developing CA3 Hippocampal Pyramidal Neurons Journal of Neuroscience 30: 6776-6781.
18. White AM, Swartzwelder HS (2206) Hippocampal Function during Adolescence: A Unique Target of Ethanol Effects. Ann N Y Acad Sci 1021: 206-220.

19. Boileau I, Assaad JM, Pihl RO (2003) Alcohol promotes dopamine release in the human nucleus accumbens. Sinapse 49: 226-231.

20. Yoder KK, Constantinescu CC, Kareken DA (2007) Heterogeneous Effects of Alcoho on Dopamine Release in the Striatum: A PET Study. Alcohol Clin Exp Res 31: 965-973.

21. Volkow ND, Wang GJ, Telang F (2007) Profound Decreases in Dopamine Release in Striatum in Detoxified Alcoholics: Possible Orbito-frontal Involvement. J Neurosci 27 : 12700-12706.

22. Yoshimoto K, McBride WJ, Lumeng L, Li TK (1992) Ethanol Enhances the Release of Dopamine and Serotonin in the Nucleus Accumbens of HAD and LAD Lines of Rats. Alcohol Clin Exp Res 16: 781-785.

23. Richter CP, Campbell KH (1940) Alcohol taste thresholds and concentrations of solution preferred by rats. Science 91: 507-508. [Crossref]

24. Shoaib M, Almeida O (1996) Absence of tolerance to the eversive stimulus properties of ethanol following oral ethanol self-administration. Alcohol 2: 175-180.

25. Bull D, Palij M, Millar J (1990) Application of fast cyclic voltammetry to measurement of electrically evoked dopamine overflow from brain slices in vitro. $J$ Neurosci Methods 32: 37-44.

26. White AM, Scott Swartzwelder H (2005) Age-Related Effects of Alcohol on Memory and Memory-Related Brain Function in Adolescents and Adults. In: Galanter M, Lowman C, Boyd GM, Faden VB, Witt E, et al. (Eds) Recent Developments in Alcoholism. Recent Developments in Alcoholism (Alcohol Problems in Adolescents and Young Adults), vol 17. Springer, Boston, MA.

27. Beinfeld MC, Palkovits M (1981) Distribution of cholecystokinin (CCK) in the hypothalamus and limbic system of the rat. Neuropeptides 2: 123-129.

28. Palkovits M (2013) Topography of chemically identi?ed neurons in the central nervous system. Neuroendocrine Perspectives 3: 1-398.

29. Crespi F, Martin KF, Heal DJ, Marsden CA, Buckett WR, et al. (1989) Measurement of 3-methoxytyramine by in vivo voltammetry: evidence for differences in central dopamine function in BALB/c and CBA mice. Brain Res 500: 241-246. [Crossref]

30. Sanghera M, Crespi F, Martin K (1990) Biochemical and in vivo voltammetric evidence for difference in striatal dopamine levels in inbred strains of mice. Neuroscience 39: 649-656.

Copyright: (C2019 Crespi F. This is an open-access article distributed under the terms of the Creative Commons Attribution License, which permits unrestricted use, distribution, and reproduction in any medium, provided the original author and source are credited. 